\title{
An analysis of two-layer models for circumstellar disks
}

\author{
C. P. Dullemond ${ }^{1}$ and A. Natta ${ }^{2}$ \\ 1 Max Planck Institut für Astrophysik, Postfach 1317, 85741 Garching, Germany \\ 2 Osservatorio Astrofisico di Arcetri, Largo E. Fermi 5, 50125 Firenze, Italy
}

Received 17 February 2003 / Accepted 15 April 2003

\begin{abstract}
The two-layer disk models of Chiang \& Goldreich (1997, henceforth CG) and its derivatives are popular among astronomers because of their simplicity and the clear predictions they make for the SEDs of T Tauri stars and Herbig Ae/Be stars. Moreover, they can be computed quickly, which is a great advantage when fitting observations using automated procedures. In this paper we wish to assess the accuracy and reliability of 2-layer models, by comparing them to detailed vertical structure models with accurate 1+1D radiative transfer. We focus on the shape of the SED, and the predicted height and "flaring index" of the disk. We first consider models where scattering is set to zero. We find that 2-layer models overestimate significantly the near-infrared flux, and we suggest a simple way of correcting this effect, at least in part. At longer wavelengths, the SED of twolayer models often show a two-bump structure, which is absent in 1+1D models. Nevertheless, overall agreement is reasonably good, and the differences are in most cases within 30\%. At (sub)-mm wavelengths the differences may even be less. The shape of the disk, as measured by its pressure and surface scale height and by the flaring angle are also well reproduced by two-layer models. When scattering is included in the 1+1D models, the differences become larger, especially in the near-infrared. We suggest simple ways to include scattering in two-layer models and discuss their reliability. We do not compare the two-layer models to full 2D/3D models, so the conclusions remain valid only within the annulus-by-annulus approximation.
\end{abstract}

Key words. accretion, accretion disks - stars: circumstellar matter - stars: formation - stars: pre-main-sequence infrared: stars

\section{Introduction}

Models of disks around pre-main-sequence stars are currently used to make predictions of a number of observable quantities and to compare them to observations. The complexity of such models varies from so-called power-law disks, where all the relevant disk properties are described by powerlaw of radius and changed independently (see, for example, Beckwith et al. 1990), to models where the thermal and geometrical properties are computed self-consistently, under the assumption of hydrostatic equilibrium (D'Alessio et al. 1998; Malbet et al. 1991, 2001; Bell et al. 1997, 1999; Dullemond et al. 2002). A large effort is currently being made in improving the treatment of radiation transfer, by developing $2 \mathrm{D}$ codes that deal efficiently with the very large optical depths that characterize such disks (e.g. Bjorkman \& Wood 2001; Dullemond 2002). At the same time, it is clear that the need of simple models, that can be easily used in interpreting the observations, remains. This explain the success of the two-layers disk schematization proposed some years ago by Chiang \& Goldreich (1997; henceforth CG97).

Because the CG97 model and its derivatives (e.g. Chiang et al. 2001; Dullemond et al. 2001; Lachaume et al. 2003)

Send offprint requests to: C. P. Dullemond, e-mail: dullemon@mpa-garching .mpg.de are often used in interpreting disk observations, it is important to estimate their reliability and accuracy by comparing them to more detailed and self-consistent model calculations. At present, self-consistent 2D and 3D models are still too cumbersome for such a comparison. However, there are a number of 1D vertical structure models based on detailed radiative transfer, which can be used as templates to assess the reliability of 2-layer models.

In this paper, we will make use of the model of Dullemond et al. 2002 (henceforth DZN02). In this model the vertical temperature profile was solved using a detailed plane-parallel 1D vertical radiative transfer procedure, which treats properly the slanted penetration of the stellar radiation (often referred to as $1+1 \mathrm{D}$ approximation). Using the resulting temperature profile, the density structure is re-computed after each iteration step by integration of the equation of vertical hydrostatic equilibrium. In a follow-up paper (Dullemond \& Natta 2003) scattering of the stellar light by the dust particles was included in the model. For the case of zero albedo this model is in fact identical to the DZN02 model, and we will use DN03 as our "standard 1+1D disk structure model" against which we will compare the two-layer models.

The goal of this paper is three-fold. First, and most importantly, we compare an improved version of the CG97 model to the the 1+1D vertical structure model of DN03. 
This initial comparison is done for dust opacities in which scattering is switched off. Then we investigate how big the effects of scattering are in comparison to the typical errors of the improved CG model. Finally we discuss the possibility of using a simple recipe to include scattering into the CG model.

\section{Description of the models}

In this section we give a short description of the improved CG97 model we adopt, and of the $1+1 \mathrm{D}$ vertical structure model. Both families of models neglect viscosity, and treat the radial dependence of the surface density as a free parameter. Consequently, they also do not include viscous heating which, depending on the value of the viscosity parameter $\alpha$, could be of importance in the inner parts of the disk.

\subsection{Improved CG97 model ("CG+")}

The central element of the two-layer Chiang and Goldreich model is that, due to the very shallow incident angle $\alpha \ll 1$, the impinging stellar radiation is absorbed entirely in the very tenuous upper layers of the disk, high above the disk's photosphere. While this layer has an optical depth of unity for stellar radiation at that grazing angle (typically around $\alpha=0.05$ ), it is very optically thin in the vertical direction $\left(\tau_{\text {vertical }} \simeq \alpha\right)$. Energy conservation requires that this absorbed radiation is re-emitted as infrared radiation, half of which is emitted away from the disk, while the other half is emitted towards the disk midplane. This downwards emitted flux is absorbed by the disk interior and re-emitted once more in the infrared, though this time at longer wavelengths. This typically gives two components in the SED: a warm optically thin component with dust emission features in the near- to mid infrared and a cool thermal blackbody component from the disk interior at longer wavelengths. If the disk is optically thick to the radiation from the midplane, then both components are equally strong, as the cool component is a reprocessed version of the downward radiated surface emission. The temperature $T_{\mathrm{s}}$ of the surface layer is fixed: it is the optically thin dust temperature. The emissivity of the surface layer is determined by the surface density of the surface layer, which is calculated from energy conservation: the total emission should equal the total absorbed flux. The emission from the interior is determined by the temperature of the disk interior and the disk's surface density. In the simple case of a grey opacity and high optical depth this so-called midplane temperature is determined by equating the thermally emitted flux $F=\sigma T_{i}^{4}$ to the downwards directed surface flux. In general, $T_{i}$ is computed using mean opacities both for the heating and for the cooling radiation.

The original paper of Chiang \& Goldreich (1997) describes the two-layer flaring disk model in stages. At first, a grey opacity constant surface density model is presented for which the flaring index $\mathrm{d} \log \left(H_{\mathrm{S}}\right) / \mathrm{d} \log (R)=9 / 7$, where $H_{\mathrm{s}}$ is the height of the surface. Then a set of improvements are listed in order to account for effects of low optical depth at various wavelength regimes. In a later paper (Chiang et al. 2001) the model was refined to include more realistic opacities and, more importantly, the self-consistent (and numerically stable) determination of the flaring angle, which is crucial for energy conservation. Dullemond et al. (2001, henceforth DDN01) subsequently added the emission and structure of the disk's inner rim, and included effects of self-irradiation and self-shadowing. The complete set of model equations is listed in DDN01. In the rest of this paper this improved Chiang \& Goldreich model will be referred to as the $\mathrm{CG}^{+}$model. We will not address here issues related to the inner rim.

\subsection{Vertical structure models}

The reference model against which we compare the $\mathrm{CG}^{+}$model is the $1+1 \mathrm{D}$ vertical structure model of DZN02 and its improved version DN03. The radiative transfer is done in a twostage procedure: first the primary stellar photons are followed as they penetrate the surface layers of the disk and get absorbed. Then the re-emitted infrared radiation is solved using a 1D vertical frequency and angle-dependent radiative transfer code applied at each radial annulus. In a third stage the vertical pressure balance is solved, under the assumption that the gas temperature equals the dust temperature and that the gas-to-dust ratio is constant. This three-stage procedure is then iterated until a converged solution is reached for the complete temperature and density structure of the disk. During each iteration, the flaring index has to be recomputed in order to compute the flaring angle ("grazing angle" in the language of Chiang \& Goldreich) self-consistently. This is done by estimating the surface height of each annulus by finding the height $H_{\mathrm{s}}$ above the midplane where the direct stellar radiation is extincted by $\exp (-1)$. The double-logarithmic derivative of this $H_{\mathrm{s}}$ with cylindric radius $R$ is then the flaring index from which the flaring angle can be computed (see DZN02).

Once the iteration procedure has converged, we have obtained a solution for the dust temperature $T_{\text {dust }}(R, Z)$ and the disk's gas+dust density $\rho(R, Z)$.

DN03 included scattering in the following way. Stage 1 of the DZN02 model (the irradiation of the disk by primary stellar photons) was replaced with a 1D Monte-Carlo code. Primary stellar photons are followed as they penetrate into the surface of the disk and scatter around until they either get absorbed, or escape to infinity. If a photon is absorbed, it leaves behind its energy, which is then used as a source term for the radiative transfer of the second stage. In the second stage, which takes care of the re-emitted infrared radiation from the disk, scattering is not included. This is a valid approximation if the dust grains are small enough (typically not larger than $0.5 \mu \mathrm{m}$ ).

\section{Comparing models without scattering}

As a first step we compare the $\mathrm{CG}^{+}$model and the $1+1 \mathrm{D}$ vertical structure for a test case without scattering. In this way the physical assumptions of both models are the same, so that in principle we expect both models to produce very similar SEDs. The comparison is first done for a single annulus at various radii and optical depths, irradiated by a $\mathrm{T}$ Tauri star or by a Herbig Ae/Be star. We then compare the SEDs of models of entire disks, as well as intensity profiles at some selected wavelengths. 


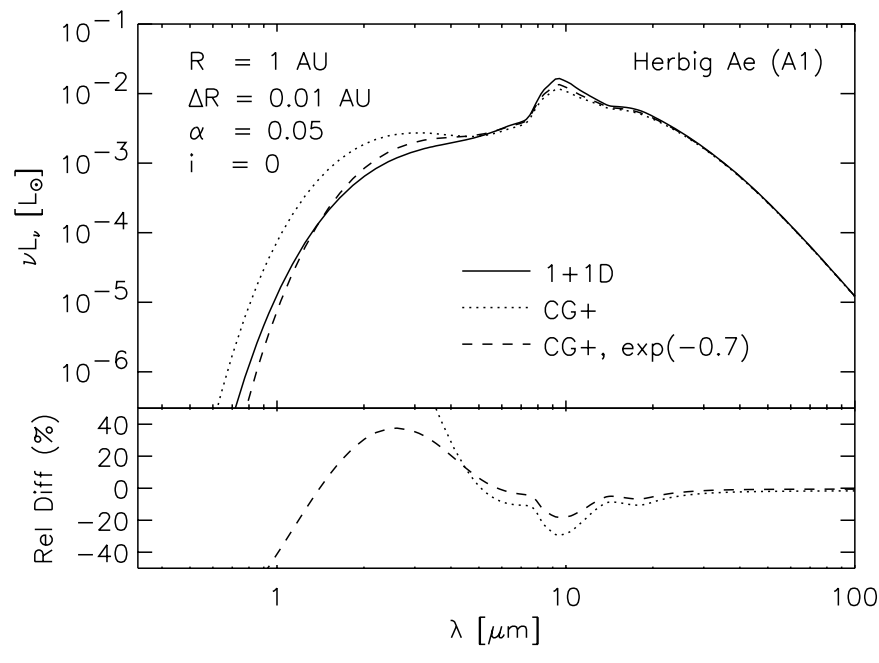

Fig. 1. The SED for the single-annulus test case nr. A1 (Herbig Ae star) at $1 \mathrm{AU}$, compared to the $\mathrm{CG}^{+}$model in its two proposed variants.

\subsection{Single annulus}

As a first example we consider an annulus of a disk at $1 \mathrm{AU}$ from the central star, with width $0.01 \mathrm{AU}$ (i.e. an annulus between 1.00 and $1.01 \mathrm{AU})$. We take the central star to be a Herbig Ae/Be star $\left(M_{*}=2 M_{\odot}, R_{*}=2 R_{\odot}, T_{\text {eff }}=10000 \mathrm{~K}\right)$. To simplify matters, we assume the spectrum of the star to be a blackbody spectrum with $T=T_{\text {eff }}$. The grazing angle (i.e. the angle at which the stellar radiation enters the disk's atmosphere) is computed self-consistently in both $\mathrm{CG}^{+}$and $1+1 \mathrm{D}$ models, but for the purpose of the comparison described in this section we assume it to be fixed to $\alpha=0.05$. We also assume, for these annulus tests, that half of the surface of the star as seen from the top of the annulus is covered by the very inner parts of the disk (see CG97). This effectively reduces the stellar flux by a factor 0.5 . The absorption cross section is that of a silicate grain of $0.1 \mu \mathrm{m}$ (Draine \& Lee 1984), with zero albedo. Different choices of the opacity will not change the results of this paper in any significant way, unless explicitely noted. The surface density of the disk is such that the total vertical absorption optical depth (from $z=-\infty$ to $z=\infty$ ) at $550 \mathrm{~nm}$ is $\tau_{550 \mathrm{~nm}}=10$. The mean molecular weight is assumed to be $\mu=2.3$.

In Fig. 1 the SED of this annulus is shown for both the vertical structure model and the $\mathrm{CG}^{+}$model. The difference between the models is less than $30 \%$ at wavelengths $\lambda>8 \mu \mathrm{m}$. At shorter wavelengths the $\mathrm{CG}^{+}$model has a bump that is not present in the SED of the vertical structure model. The reason can be traced back to the surface temperature assumed in the $\mathrm{CG}^{+}$model, which is that of grains in an optically thin medium. In reality, however, only a fraction of the grains in the surface layer see the unextincted stellar light. More than $36 \%$ of the material in the surface layer lies at optical depths larger than unity (along the grazing incident path of the stellar radiation). It is hard to assign a single temperature to the material in the surface layer. But a reasonable average temperature could be the temperature at an optical depth of $\tau=0.7$, which is the location where exactly half of the stellar radiation has been

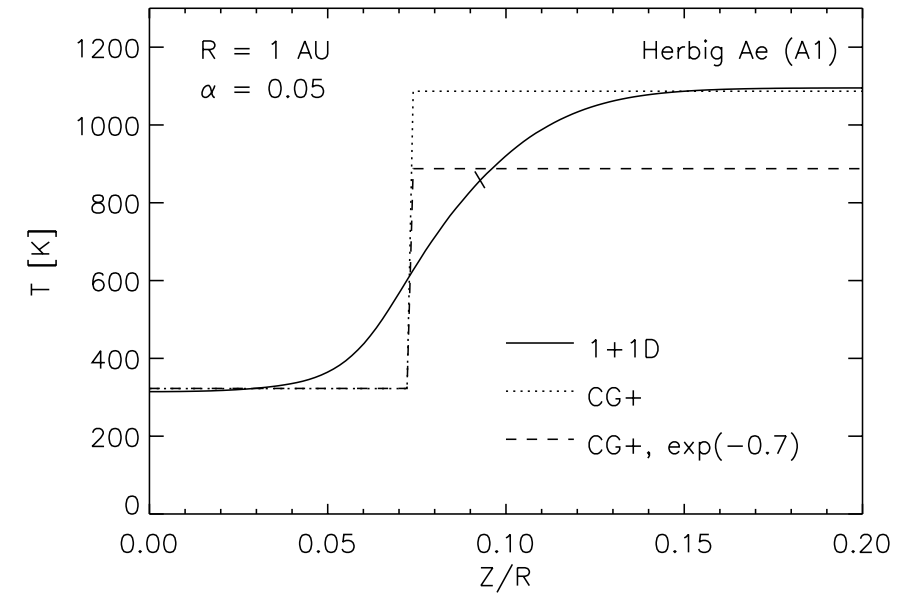

Fig. 2. The temperature structure for the single-annulus test case A1 (Herbig Ae star), compared to the $\mathrm{CG}^{+}$model in its two proposed variants. The transition from the surface to the midplane temperature in the $\mathrm{CG}^{+}$models occurs at $H_{\mathrm{s}}$, i.e., where the optical depth along a ray at a grazing angle $\alpha=0.05$ at stellar wavelengths is $\tau_{*} \sim 1$. The tick mark on the solid curve shows the location of $H_{\mathrm{s}}$ in the $1+1 \mathrm{D}$ case.

absorbed. The dashed line in Fig. 1 shows the SED as computed by the $\mathrm{CG}^{+}$model in which the surface temperature is evaluated at an extinction of $\exp (-0.7)=0.5$.

In Fig. 2 the temperature structure of the same annulus is shown as a function of the vertical coordinate $Z / R$, where $Z$ is the height above the midplane, and $R$ the radius from the star (in this case $R=1 \mathrm{AU}$ ). High above the surface of the disk the temperature is constant, and virtually equal to the optically thin dust grain temperature at that distance from the star. The slight difference is due to the fact that in the $1 \mathrm{D}$ model the dust high above the disk is heated not only by the direct stellar radiation but also by the thermal emission of the disk below it.

As one goes towards smaller $Z$ the temperature starts to decrease due to extinction of the direct stellar flux by the material of the disk. This is the location of the disk's "hot surface layer" which produces the dust emission features. The small tick mark on the temperature curve denotes the surface height $H_{\mathrm{s}}$ where the grazing optical depth is unity, i.e. where most of the direct stellar radiation is absorbed. Continuing downwards from the surface layers, the temperature slope levels off again because the disk's own infrared radiation becomes competitive with the direct stellar radiation in the thermal balance equation of the dust grains. This is where the disk's photosphere is located and where most of the thermal continuum emission is produced (the midplane emission, in the terminology of CG models).

Over-plotted over the smooth temperature curve is the twolayer $\mathrm{CG}^{+}$model, in its two variants mentioned above. The sharp step in the temperature profile is where the surface layer of the $\mathrm{CG}^{+}$model starts: $z=H_{\mathrm{s}}$. The main difference in the two variants of the model is the temperature of this surface layer. For one variant, the surface layer temperature equals that of optically thin dust grains. For the corrected variant this temperature is lower, and is closer to the temperature of the vertical structure model at the location of the tick mark.

Note that $H_{\mathrm{s}}$ is different in $\mathrm{CG}^{+}$and $1+1 \mathrm{D}$ models. Most of difference is due to the fact that in the $1+1 \mathrm{D}$ models the density 
Table 1. The parameters of the single-annulus models used in the comparison. The first column is the identification used in this paper. Columns 2-4 are the stellar parameters (in units of $M_{\odot}, R_{\odot}$ and $\mathrm{K}$ ) and Cols. 5-6 are the annulus parameters. The radius is in AU. All model annuli have a width $\Delta R / R=0.01$ and a grazing incident angle of stellar radiation of $\alpha=0.05$.

\begin{tabular}{cccccc}
\hline \hline & $M_{*}$ & $R_{*}$ & $T_{*}$ & $R$ & $\tau_{V}$ \\
\hline A1 & 2 & 2 & 10000 & 1. & 10 \\
A2 & 0.5 & 2 & 4000 & 0.1 & 10 \\
A3 & 2 & 2 & 10000 & 50. & 300 \\
\hline
\end{tabular}

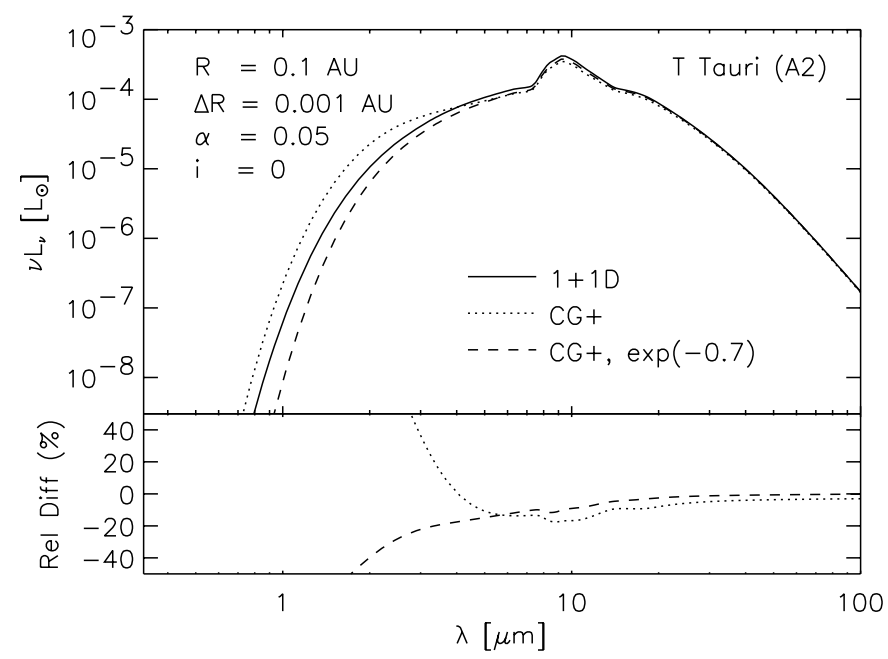

Fig. 3. The SED for the single-annulus test case No. A2 (T Tauri star) at $1 \mathrm{AU}$, compared to the $\mathrm{CG}^{+}$model in its two proposed variants.

in the disk surface deviates from the Gaussian shape predicted by isothermal models (see, for example, Fig. 3 of DN03). An additional, smaller difference is due to the fact that $\mathrm{CG}^{+}$models use the mean opacity averaged over the stellar flux to define $H_{\mathrm{s}}$, while the 1+1D models use the wavelength-dependent opacity.

Table 1 lists the parameters of two other annuli. Test A2 is the T Tauri analog of A1, with lower stellar mass and temperature, and the annulus taken at a smaller radius (to obtain similar temperatures in the disk). In Fig. 3 the SED of this test case is shown. The results are qualitatively similar to the case of the Herbig Ae star (test A1). In this particular example, however, the $\exp (-0.7)$ correction of the surface temperature calculation in the $\mathrm{CG}^{+}$model does not make a major improvement, although also in this case the overall SED of the $1+1 \mathrm{D}$ model is slightly better reproduced when this factor is taken into account.

As shown above, in many cases the $\mathrm{CG}^{+}$model is reasonably well in agreement with the $1+1 \mathrm{D}$ vertical structure model. Yet, there are cases in which the differences are appreciable. We show an example in Fig. 4 which plots the SED of the A3 annulus. This annulus has $\tau_{V}=300$, and is at $50 \mathrm{AU}$ from our Herbig Ae star. It is clearly seen that in this case the difference between $\mathrm{CG}^{+}$and $1+1 \mathrm{D}$ predictions is rather large, though still within a factor of 2 . The explanation for the breakdown of the $\mathrm{CG}^{+}$model in this case is likely the use of mean opacities to

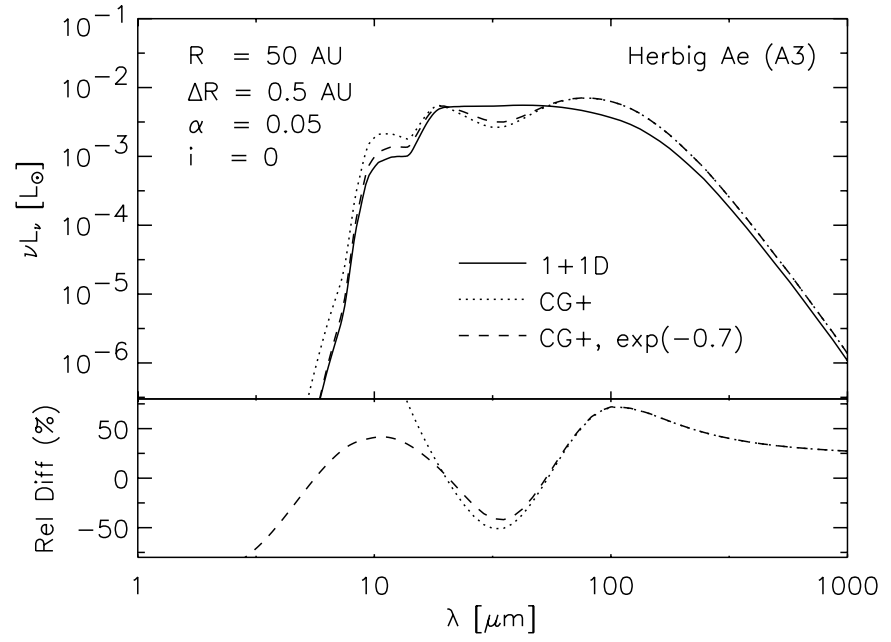

Fig. 4. The SED for the single-annulus test case No. A3 (Herbig Ae star, high optical depth) at $50 \mathrm{AU}$, compared to the $\mathrm{CG}^{+}$model in its two proposed variants.

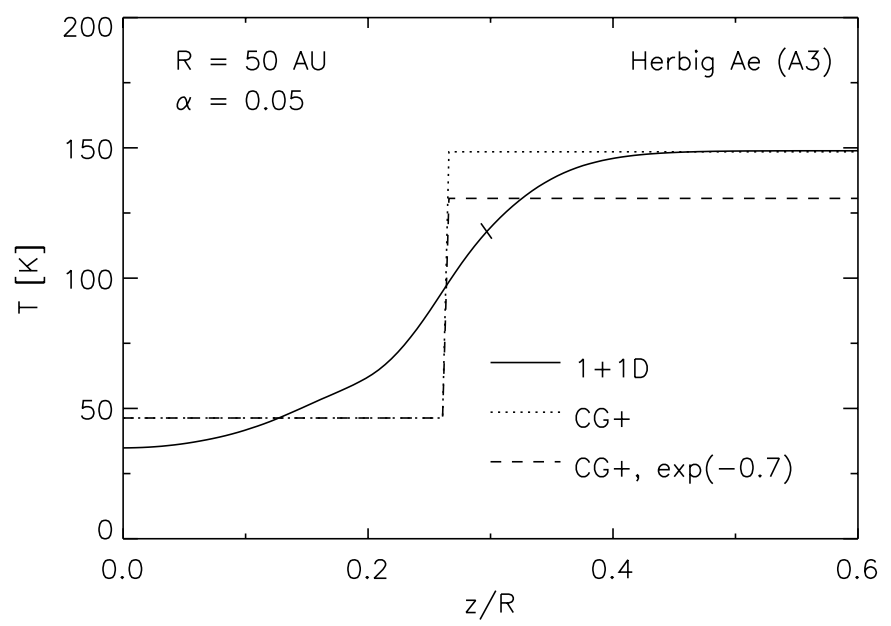

Fig. 5. The temperature structure for model A3.

compute the midplane temperature. As noted by DZN02, the midplane of the disk can cool down by leaking radiation at long wavelengths (where the optical depth of the disk becomes smaller than unity), more than expected on the basis of a meanopacity analysis. This is clearly seen in Fig. 5, which shows the temperature structure of the A3 annulus. The $1+1 \mathrm{D}$ temperature profile, which goes from values lower than predicted by $\mathrm{CG}^{+}$in the midplane to larger ones at intermediate $z$ results in lower fluxes around $100 \mu \mathrm{m}$ and higher fluxes in the mid-infrared, so that the two-bump shape of $\mathrm{CG}^{+}$practically disappears in the 1+1D model.

\subsection{Full disk models}

In this section we compare the SED and the physical structure of entire disks computed with the $1+1 \mathrm{D}$ vertical structure model and $\mathrm{CG}^{+}$models. We present three illustrative cases: a disk around a Herbig Ae star, a T Tauri star and a Brown Dwarf. Table 2 lists the parameters of the three disk models. 
Table 2. The parameters of the full disk models. The first column is the identification used in this paper. Columns $2-4$ are the stellar parameters (in units of $M_{\odot}, R_{\odot}$ and $\mathrm{K}$ ) and Cols. 5-6 are the annulus parameters: outer radius in $\mathrm{AU}$, and the surface density $\Sigma$ at $1 \mathrm{AU}$. All models have a power law index for the surface density of -1 ( $\Sigma \propto$ $1 / R$ ). Column 8 is the mass of the disk in units of $M_{\odot}$ as derived from the other parameters.

\begin{tabular}{cccccccc}
\hline \hline & $M_{*}$ & $R_{*}$ & $T_{*}$ & $R_{\text {in }}$ & $R_{\text {out }}$ & $\Sigma(1 \mathrm{AU})$ & $M_{\text {disk }}$ \\
\hline F1 & 2 & 2 & 10000 & 1 & 300 & 1000 & 0.2 \\
F2 & 0.5 & 2 & 4000 & 0.1 & 300 & 100 & 0.02 \\
F3 & 0.1 & 1.3 & 2600 & 0.033 & 30 & 100 & 0.002 \\
\hline
\end{tabular}

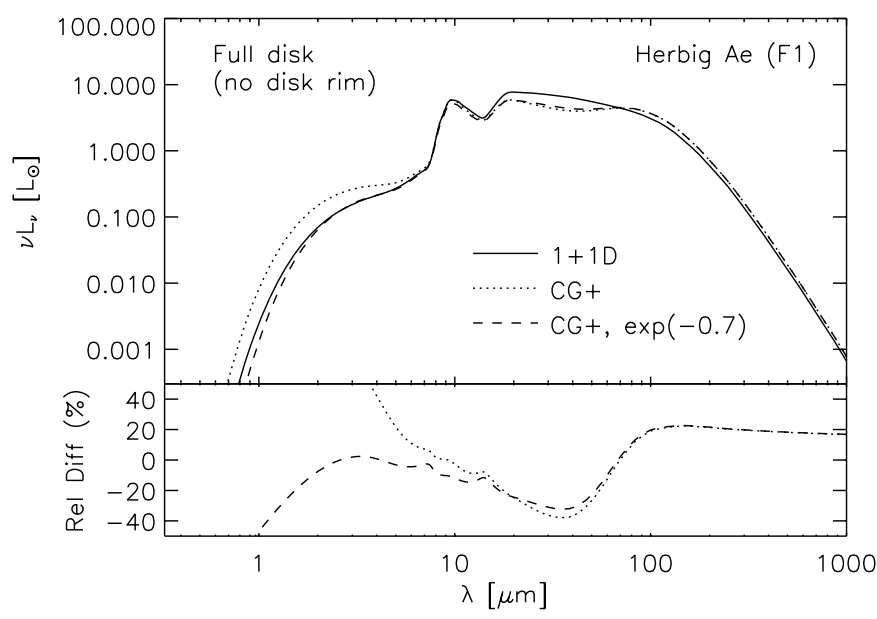

Fig. 6. The SED for the full disk model F1 (Herbig Ae star), compared to the $\mathrm{CG}^{+}$model in its two proposed variants.

We assume that the disk is in hydrostatic equilibrium and compute the flaring angle is self-consistently. This is done by evaluating the flaring index

$\xi \equiv \frac{\mathrm{d} \lg \left(H_{\mathrm{s}} / R\right)}{\mathrm{d} \lg R}$,

where $H_{\mathrm{s}}$ is the height of the $\tau_{\text {grazing }}=1$ surface of the disk. From the flaring index, the flaring angle can be computed directly:

$\alpha=0.4 \frac{R_{*}}{R}+\xi \frac{H_{\mathrm{s}}}{R}$.

For numerical stability reasons this flaring index is always evaluated 2 grid points inwards of the point where it is used (see Chiang et al. 2001). In these models we assume that the stellar flux is not reduced due to occultation of the central star by the inner disk, as was assumed in the single-annulus models.

Figure 6 shows the SED of model F1. Over-plotted are the two proposed $\mathrm{CG}^{+}$models (the one with optically thin surface temperature and the one with the correction factor $\exp (-0.7)$ ). One can see that overall the models agree, but that the $\mathrm{CG}^{+}$ model clearly shows a double-bump structure between $20 \mu \mathrm{m}$ and $100 \mu \mathrm{m}$ which is not seen in the full vertical structure model. In Fig. 7 the same is plotted, but now for a T Tauri star (model F2). The disk is taken to be less massive than the Herbig star disk. The $\mathrm{CG}^{+}$model works really well for this case, with

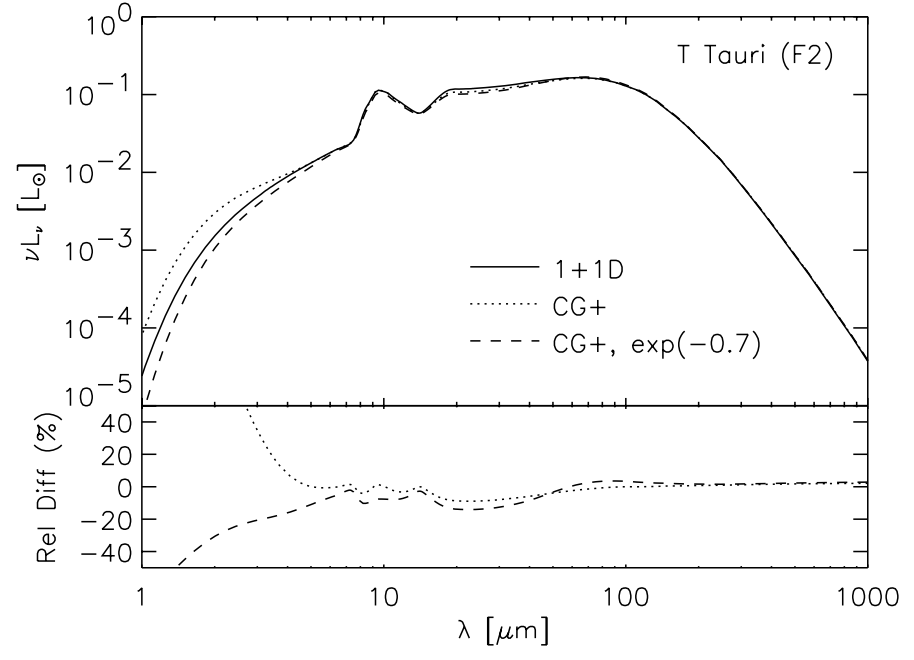

Fig. 7. Same as Fig. 6, but now for model F2 (a T Tauri star).



Fig. 8. Same as Fig. 6, but now for model F3 (a Brown Dwarf).

errors mostly within $10 \%$. Finally, in Fig. 8 the SED of a Brown Dwarf disk model is show (model F3). This disk is taken to have a much smaller outer radius and mass than the $\mathrm{T}$ Tauri case. Nevertheless, the disk remains very optically thick, as in the other models. Again, errors are reasonably small, though slightly larger than for model F2.

In Fig. 9 the height of the disk of model F1 (Herbig Ae star) and F2 (the T Tauri case) is shown as a function of radius, for both the $1+1 \mathrm{D}$ model and the $\mathrm{CG}^{+}$model. The pressure scale height is derived from the temperature of the disk at the equatorial plane. At very small radii $H_{\mathrm{p}}$ is virtually the same in $\mathrm{CG}^{+}$ and $1+1 \mathrm{D}$ models. At larger radii, $H_{\mathrm{p}}$ in the $1+1 \mathrm{D}$ model drops below that of the $\mathrm{CG}^{+}$model, because the equatorial plane temperature of the 1+1D model tends to be lower, as discussed in Sect. 3.1. The difference, however, is always very small.

The surface scale height in $H_{\mathrm{s}}$ tends to be larger in $1+1 \mathrm{D}$ models, reflecting in part the deviation of the very upper layers from a Gaussian profile, as discussed of Sect. 3.1. The degree by which $\mathrm{CG}^{+}$models can reproduce the results of $1+1 \mathrm{D}$ ones depends on the dust opacity in combination with other model parameters, such as the disk mass. Here we just 



Fig. 9. Top panel: pressure scale height $H_{\mathrm{p}}$ and surface height $H_{\mathrm{s}}$ as function of $R$ for model F1 (Herbig Ae star). The solid line shows the results of the vertical structure model, the dashed line those of $\mathrm{CG}^{+}$ones. Bottom Panel: same for model F2 (T Tauri star).

note that the difference is larger for the HAe star, and for larger radii. However, these differences remain small and we can conclude that, in all cases we have considered, the disk shape can be derived with good accuracy using the simple approximations of the $\mathrm{CG}^{+}$models.

Figure 10 shows the flaring index as function of radius for model F2. This quantity gives information on the amount of radiation emitted by the disk at any given radius, because for $R \gg R_{*}$ the emission is proportional to $\xi$. Although the run of $\xi$ with $R$ is similar in the two models, we note that $\xi$ is a smooth function of radius in the $\mathrm{CG}^{+}$models, but not in the $1+1 \mathrm{D}$ one. In particular, the kink in the flaring index $0.3 \mathrm{AU}$ is not due to numerical inaccuracies but rather to the behavior of the midplane optical depth, which in this disk model drops below unity at long wavelengths at about that radius. The consequent cooling of the midplane below optically thick temperatures, discussed in Sect. 3.1, is seen as a slight kink in $H_{\mathrm{s}}$ in Fig. 9. This effect, which depends on the disk and stellar parameters and on the exact wavelength dependence of the dust opacity, can have important consequences on the stability of $1+1 \mathrm{D}$ models.

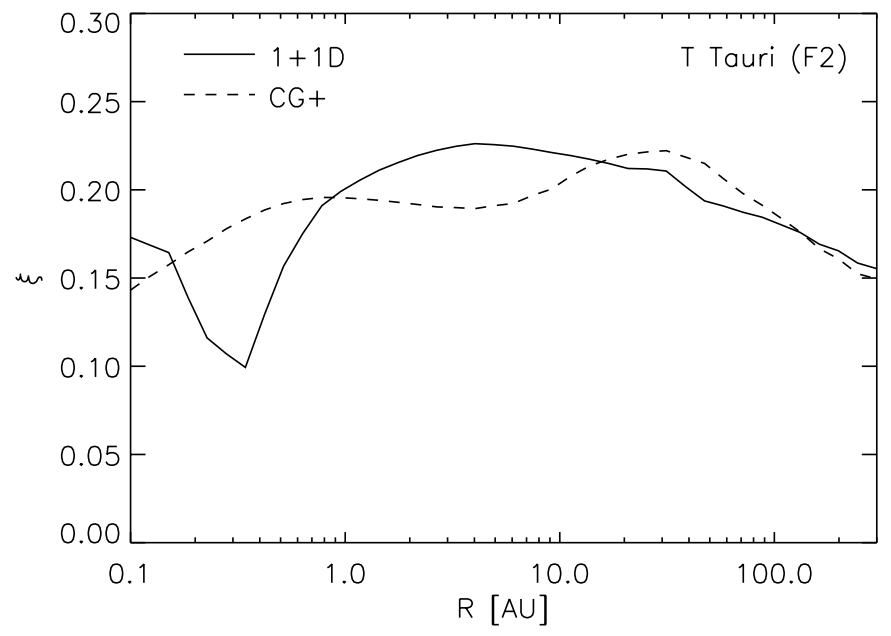

Fig. 10. The flaring index of the disk $\xi$ is plotted as a function of radius for model F2 (T Tauri star). The vertical structure model is shown as a solid line, while the $\mathrm{CG}^{+}$is the dashed line.

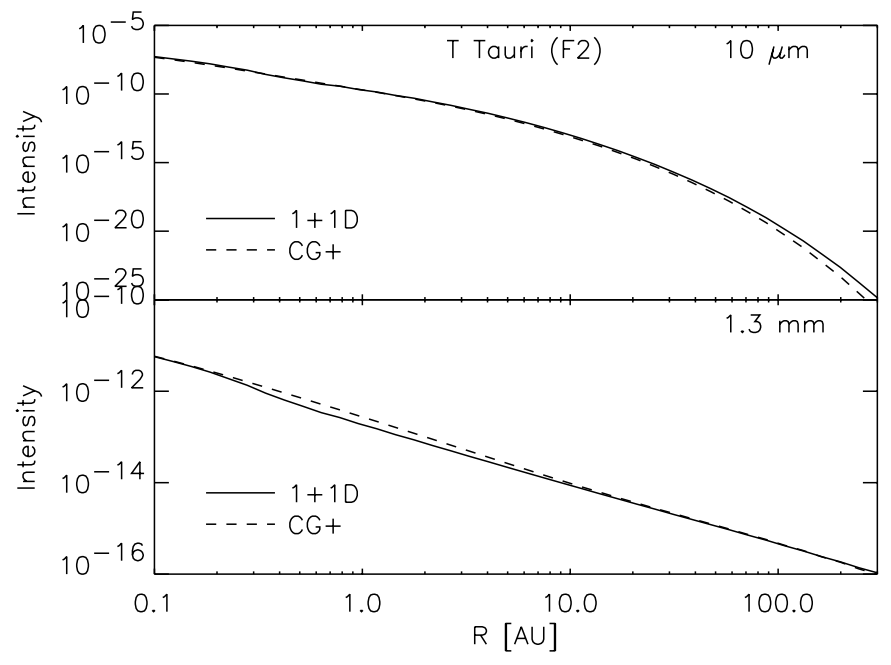

Fig. 11. The brightness profile of model F2 (T Tauri star) as a function of radius at two different wavelengths. The 1+1D model results are shown as a solid line, the $\mathrm{CG}^{+}$model results as a dashed line.

Figure 11 shows the brightness profiles of the two model approaches at two wavelengths, $10 \mu \mathrm{m}$ and $1.3 \mathrm{~mm}$, where current instrumentation has the spatial resolution required to resolve the disks. The differences between the models are very small, probably within the observational accuracy of most experiments. The $10 \mu \mathrm{m}$ profile has a clear exponential cut-off at large radii due to the fact that the dust becomes too cool to emit strongly at mid-infrared wavelengths. The $\mathrm{CG}^{+}$and $1+1 \mathrm{D}$ models produce virtually identical emission at this wavelength, as seen also in the SED (Fig. 7). At $1.3 \mathrm{~mm}$ the differences are slightly larger (up to $30 \%$ ), which reflects the fact that sometimes the $1+1 \mathrm{D}$ models can be slightly cooler in the disk's midplane than the $\mathrm{CG}^{+}$models, as discussed above. 




Fig. 12. Example of the effect of scattering on the SED of a T Tauri disk (model F3). Only the thermal emission of the disk is shown in this figure. Scattered light would appear as a strong rise towards short wavelengths.

\section{Models with scattering}

\subsection{Effect of scattering on the disk}

The effects of scattering of the stellar radiation on the structure and SED of circumstellar disks was discussed in detail in DN03. The main effect is that the thermal infrared flux from the disk is reduced at all wavelengths, with a larger effect at shorter than at longer wavelengths. This reduction takes place because the scattering reflects away from the disk part of the stellar radiation, which therefore cannot be reprocessed into infrared radiation. The reflected fraction of the incident flux can be evaluated analytically using the H-functions of Chandrasekhar, which were discussed in this context in DN03. For a moderate value of the albedo $(\omega \sim 0.5)$ and isotropic scattering the reflected fraction is about $25 \%$ (of order $\omega / 2$ ), and the reduction factor of the disk's thermal emission is about 0.75 , when averaged over the infrared spectrum. For higher albedo the reduction is larger, while it decreases if the scattering is more forward-peaked.

The reason why the reduction is stronger at short wavelength than at long wavelengths can be traced back to the fact the surface layers of the disk are affected more strongly by scattering than the interior. A photon that gets lost in the disk's interior may scatter many times and still get absorbed rather than escape. Therefore the deep interior layers of the disk will have a temperature only slightly below the temperature of a disk without scattering.

The SEDs of a T Tauri disk (model F2) with increasing albedo, computed as in DN03, are shown in Fig. 12. The effect of reddening of the SED as $\omega$ increases is clearly seen. This suggests that $\mathrm{CG}^{+}$model-predicted SEDs, which tend in general to overestimate the amount of flux at shorter wavelengths with respect to $1+1 \mathrm{D}$ models, may be significantly in error when realistic dust models are considered.

\subsection{A simple recipe for scattering in $\mathrm{CG}^{+}$models: Is it possible?}

Although the qualitative effect of scattering can be easily understood "a priori", it is more difficult to estimate quantitatively how the infrared spectrum of the disk is affected at each individual wavelength. Still, it is important to explore the possibility of including scattering in the $\mathrm{CG}^{+}$models in some simple way, and to assess the reliability of any such model by comparing its predictions to those of the more accurate $1+1 \mathrm{D}$ models.

A first recipe is based on the following considerations. In $\mathrm{CG}^{+}$models, the stellar radiation is intercepted by the disk surface, which will re-emit (part as scattered light and part as reprocessed radiation) $1 / 2$ of it toward the observer and $1 / 2$ toward the midplane. This entire second $1 / 2$ will be absorbed by the midplane, whose temperature, therefore, will not depend on $\omega$. Of the $1 / 2$ emitted toward the observer, a fraction $\omega$ is in the form of scattered light, and consequently only a fraction $(1-\omega)$ is in the form of thermal reprocessed flux at infrared wavelength. Since the temperature of this surface layer dust depends only on its absorption cross section, one can compute the SED by combining the emission of the midplane of a $\mathrm{CG}^{+}$model with $\omega=0$ with the surface emission reduced by a factor $(1-\omega / 2)$. This recipe neglects multiple scattering and any wavelength-dependent effect. However, for low albedo it reproduces well the overall reduction of the infrared excess, and it is based on very simple arguments.

A second possibility, suggested also by CG97, is to apply the classical result that the fraction of incident photons absorbed by a plane-parallel slab is reduced by a factor $\eta=$ $\sqrt{1-\omega}$. The midplane will then have a temperature (in the optically thick case) lower than $T(\omega=0)$ by a factor $\eta^{1 / 4}=$ $(1-\omega)^{1 / 8}$. The surface grain temperature is again unchanged, but the infrared emission of the surface needs to be reduced by a factor $\eta$. For not too high albedo, also this recipe will reproduce the overall reduction of the infrared excess found by DN03.

We show a comparison of these two prescriptions with the correct results of DN03 in Fig. 13. The top panel shows the comparison for the Herbig Ae star disk (F1), the bottom panel for the T Tauri star disk (F2). Each curve shows the fractional difference of the SED (reprocessed radiation only) of $\mathrm{CG}^{+}$models, as labeled, to that of the comparison model, which is the $1+1 \mathrm{D}$ one with $\omega=0.5$ (isotropic scattering). The solid line shows the results for $\mathrm{CG}^{+}$models where scattering is ignored. One can see that these models overestimate the flux at all wavelengths, and that the discrepancy can be larger than a factor of two (for the Herbig Ae disk) between about 2 and $5 \mu \mathrm{m}$. At longer wavelengths the agreement improves, and becomes quite good below about $100 \mu \mathrm{m}$.

The dashed and dotted lines show the results obtained with the $\mathrm{CG}^{+}$model using recipe 1 and recipe 2 , respectively. Both prescriptions seem to work equally well, and improve the SED description at wavelengths longer than about $2-3 \mu \mathrm{m}$, reducing the discrepancies below $\sim 20 \%$. Note that the larger difference in the Herbig Ae SED around $40 \mu \mathrm{m}$ is not related to scattering (see Sect. 3.2). Although we do not find any strong reason to prefer recipe 1 over recipe 2 , we note that recipe 2 reproduces more correctly the overall reduction of the infrared 



Fig. 13. The factor by which scattering suppresses the SED of the disk annulus of test cases A1 and A2 for an albedo of $\omega=0.5$. The solid line is the suppression factor from the $1 \mathrm{D}$ vertical structure model. The other lines are for the two recipes proposed for the $\mathrm{CG}^{+}$model.

excess for large values of $\omega$ (compare the run of $\eta$ with $\omega$ with the results of Fig. 1 in DN03). Finally, it is interesting to notice that recipe 1 tends to suppress the $10 \mu \mathrm{m}$ feature with respect to the continuum, while this is not the case for recipe 2 .

\section{Summary and conclusion}

In this paper we analyze the reliability of two-layer models for passive irradiated flaring dusty disks around pre-mainsequence stars by comparing their SEDs with those computed from more realistic $1+1 \mathrm{D}$ vertical structure models. The two-layer model used in this paper is called $\mathrm{CG}^{+}$, and is an improved version of the Chiang \& Goldreich model (see DDN01). The 1+1D model involves detailed 1D vertical radiative transfer which treats scattering of the primary stellar photons penetrating the surface layers of the disk (see DN03). All the models presented in this paper can be downloaded as ASCII tables from http://wWw.mpa-garching.mpg.de/ PUBLICATIONS/DATA/radtrans/cgcompare/.

We start with a comparison of the SEDs for different disk models where scattering is ignored. In general, $\mathrm{CG}^{+}$models over-predict the emission at short wavelengths. This is caused by the assumption that the surface grains have the temperature of grains in an optically thin medium. In many cases one can obtain a better agreement by computing the surface temperature with at an attenuation $\exp (-0.7)$ of the stellar flux. At longer wavelengths (mid and far-infrared), the $\mathrm{CG}^{+}$SEDs sometimes shows two maxima, due to the two components of the model: one at mid-infrared wavelength (surface layer) and one at far infrared wavelengths (interior) (see, for example, Fig. 6). The 1+1D model, on the other hand, always produces a smooth SED (with the exception of dust features), which looks qualitatively somewhat different. However, a closer look shows that the agreement between different models is often quite good (within about $20 \%$ ), with some potential problems deriving from the use of mean opacities in $\mathrm{CG}^{+}$models. In our set of models, the largest discrepancies occur for the Herbig Ae star, where they reach $\sim 40 \%$ around $40 \mu \mathrm{m}$. The shape and strength of the 10 micron emission feature is in general well predicted by the $\mathrm{CG}^{+}$models, and so is the peak-over-continuum ratio of this feature and its intensity profile. We therefore argue that also other dust features will be reasonably well reproduced by $\mathrm{CG}^{+}$models. At millimeter wavelengths, both models predict similar fluxes (within $20 \%$ at most) and intensity profiles.

A comparison of the disk physical structure is also interesting. Both the pressure scale height $H_{\mathrm{p}}(R)$, which depends on the midplane temperature, and the surface height $H_{\mathrm{s}}(R)$ predicted by the $\mathrm{CG}^{+}$model agree reasonably well with the $1+1 \mathrm{D}$ models.

Note that we have used in this paper an improved version of two-layer code, which does not make use of the analytical expressions for flaring angle and temperatures derived in CG97, but derives the disk structure self-consistently. A comparison of the CG97 analytical model with the results of 1D calculations can be found in Kraus (2003).

$\mathrm{CG}^{+}$models do not include scattering of the stellar light from dust grains. When compared to the more accurate $1+1 \mathrm{D}$ models which include scattering, even for quite low values of the albedo or rather peaked-forward scattering, we find that the differences in the SED can be larger than a factor of two in the near infrared. However, as the effect of scattering itself decreases at longer wavelengths, so do the differences between models. We have implemented two different simple recipes to our $\mathrm{CG}^{+}$code to include scattering without changing the basic idea on which two-layer models are built. Both recipes seem to improve somewhat the agreement of $\mathrm{CG}^{+}$models with the results of the $1+1 \mathrm{D}$ calculations, although recipe 2 is probably a better approximation for very large values of $\omega$. (see DN03).

In summary, the results from this paper show that $\mathrm{CG}^{+}$models compare well with the much more detailed $1+1 \mathrm{D}$ vertical structure models. These two-layer models can therefore be used with reasonable confidence to fit observed SEDs of actual objects, provided that the parameters are not stretched too far. Problems could occur, for instance, when the disks are not optically thick enough, so that the annulus-byannulus approach, on which both the $\mathrm{CG}^{+}$and the $1+1 \mathrm{D}$ models are based, breaks down. In such a case one must resort to a full 2D or even 3D treatment of the problem. A future study should find out under which circumstances such a 2D/3D approach is unavoidable, or, oppositely, when the annulus-byannulus approach (and thereby the $\mathrm{CG}^{+}$approach) is justified. 


\section{References}

Beckwith, S. V. W., Sargent, A. I., Chini, R. S., \& Guesten, R. 1990, AJ, 99, 924

Bell, K. R. 1999, ApJ, 526, 411

Bell, K. R., Cassen, P. M., Klahr, H. H., \& Henning, T. 1997, ApJ, 486, 372

Bjorkman, J. E., \& Wood, K. 2001, ApJ, 554, 615

Chiang, E. I., \& Goldreich, P. 1997, ApJ, 490, 368

Chiang, E. I., Joung, M. K., Creech-Eakman, M. J., et al. 2001, ApJ, 547,1077

D’Alessio, P., Canto, J., Calvet, N., \& Lizano, S. 1998, ApJ, 500, 411
Draine, B. T., \& Lee, H. M. 1984, ApJ, 285, 89

Dullemond, C., \& Natta, A. 2003, A\&A, submitted

Dullemond, C. P. 2002, A\&A, 395, 853

Dullemond, C. P., Dominik, C., \& Natta, A. 2001, ApJ, 560, 957

Dullemond, C. P., van Zadelhoff, G. J., \& Natta, A. 2002, A\&A, 389, 464

Kraus, M. 2003, in Open Issues in Local Star Formation, ed. J. Gregorio-Hetem, \& L. Lépine

Lachaume, R., Malbet, F., \& Monin, J.-L. 2003, A\&A, 400, 185

Malbet, F., \& Bertout, C. 1991, ApJ, 383, 814

Malbet, F., Lachaume, R., \& Monin, J.-L. 2001, A\&A, 379, 515 